\title{
INTRODUZIONE AL CONVEGNO
}

\author{
FRANCESCO CAVAGNINI (*), LUCIANO MARTINI ${ }^{(\dagger)}$
}

Questo convegno è pienamente allineato con il tema centrale che caratterizza l'Expo (Nutrire il pianeta, energia per la vita), che include tutto ciò che riguarda l'alimentazione. Questa giornata, infatti, vuole portare l'attenzione su due tra le più importanti patologie legate all'alimentazione: l'obesità e l'anoressia nervosa. Con l'intervento di esperti della materia, verranno trattati aspetti epidemiologici, patogenetici, fisiopatologici e infine clinici di queste malattie.

Nei Paesi ad elevato sviluppo economico l'obesità ha assunto negli ultimi decenni una diffusione che, dapprima definita epidemica, oggi si può ben definire pandemica. Negli Stati Uniti d'America la sua prevalenza ha ormai raggiunto il $30 \%$ della popolazione, con il $5 \%$ di individui gravemente obesi. Considerando insieme soggetti obesi (con indice di massa corporea, BMI, >30) e sovrappeso (BMI tra 25 e 30), la prevalenza arriva a superare il $50 \%$ e negli ultimi 20 anni è stata contrassegnata da un tasso di crescita del $30 \%$. Perdurando un trend di questo tipo si calcola che nel 2030 quasi un americano su due sarà obeso. Stime non lontane da queste si registrano in buona parte degli altri Paesi industrializzati. Il problema sta assumendo proporzioni allarmanti anche tra gli adolescenti, mentre si sta osservando che anche nei Paesi in via di sviluppo la prevalenza di obesità va aumentando con l'adozione di stili di vita occidentali. Ovunque, la patologia da eccesso ponderale presenta una maggiore diffusione nei ceti sociali meno abbienti e con livelli più bassi di istruzione.

E' ormai accertato che l'obesità, soprattutto se con elevata com-

(*) Laboratorio di Ricerche in Neuroendocrinologia, Istituto Auxologico Italiano, Milano, Italia. E-mail: cavagnini@auxologico.it 
ponente di grasso addominale, aumenta il rischio di malattia (patologie articolari, diabete, dislipidemie, ipertensione, malattie cardiovascolari, colecistopatie e alcune forme di cancro) e riduce l'aspettativa di vita. E' stato calcolato che per ogni $15 \mathrm{Kg}$ di peso in eccesso si ha una aumento del $30 \%$ del rischio di morte prematura. L'elevata incidenza di disabilità e il precoce ritiro dal lavoro di questi pazienti, oltre a incidere sulla loro qualità di vita, viene a gravare fortemente sulla spesa socio-sanitaria. Calcolando le perdite produttive, il sovrappeso costa agli USA il 9\% del Pil e secondo l'American Heart Association, gli USA spendono circa 190 miliardi di dollari l'anno in spese mediche legate al peso. Si comprende quindi come quella all'obesità sia oggi diventata una sfida indifferibile.

L'obesità riconosce una genesi multifattoriale, nella quale fattori ambientali interagiscono con fattori genetici e, forse, con fattori organici non ancora identificati. Essa è comunque il risultato di una alterazione del bilancio energetico con una prevalenza dell'introito sul consumo calorico e il deposito dell'energia eccedente in forma di adipe. Tra i fattori ambientali, la maggiore disponibilità di cibo, insieme al diminuito dispendio energetico conseguente alla sedentarietà, possono contribuire significativamente a questo squilibrio. D'altra parte, segnatamente per le obesità gravi a sviluppo precoce, una alterazione genetica può essere responsabile di una alterazione primaria dei complessi meccanismi che intervengono nella regolazione del comportamento alimentare. Quest'ultimo è regolato da meccanismi complessi e assai sofisticati nei quali intervengono diversi sistemi peptidergici e neurotrasmettitoriali cerebrali in grado di influenzare il senso di fame e, con azione di segno opposto, il dispendio calorico. Tipici esempi sono costituiti dal Neuropeptide Y (NPY), che stimola l'assunzione di cibo e riduce il dispendio calorico realizzando un bilancio energetico positivo (azione anabolica) e dalla leptina che esercita effetti opposti (azione catabolica). Si ritiene oggi che almeno il 10\% delle obesità gravi siano causate da alterazioni genetiche dei suddetti fattori di regolazione o dei loro recettori. Tra i due determinanti del metabolismo energetico ricordati, quello ambientale e quello genetico, si è recentemente inserito un terzo fattore attualmente in fase di intenso studio: il microbiota intestinale, noto con il nome generico di flora batterica intestinale. Si tratta di un ecosistema che abita il tratto gastroenterico, costituito da circa un trilione di microrganismi tra batteri, funghi e virus; più di 160 specie che possiedono 400 volte i geni del genoma umano (23.000) e che nell'insieme hanno un peso di 
circa $1,5 \mathrm{Kg}$. A questo insieme, definito addirittura "organo" da alcuni, vengono attribuite funzioni protettive, strutturali e metaboliche; nell'ambito di queste ultime esso potrebbe anche influenzare il bilancio energetico. La comprensione della fisiologia del microbiota ci potrebbe aiutare a capire alcune discrepanze ancora inspiegabili tra le nostre valutazioni teoriche del metabolismo e i riscontri clinici.

La comunità scientifica internazionale è impegnata in un grande sforzo per contrastare la diffusione dell'obesità, considerati anche i suoi enormi costi sociali. Purtroppo, lo straordinario avanzamento delle conoscenze derivato dalla ricerca non si è tradotto finora nello sviluppo di farmaci di grande efficacia. Pertanto, rimangono al momento fondamentali le campagne di prevenzione, dirette a promuovere corretti stili di vita inerenti all'alimentazione e all'attività fisica.

L'anoressia nervosa, indubbiamente la forma più grave tra $i$ disturbi del comportamento alimentare, è una malattia psichiatrica con ricadute organiche importanti, potenzialmente mortali, che compromettono pesantemente lo stato di salute nonché la vita sociale e professionale del paziente. La malattia si caratterizza per la marcata riduzione ponderale, una distorta percezione dell'immagine corporea e una aberrazione del comportamento alimentare finalizzata al raggiungimento di una magrezza patologica. Interessa per il $90 \%$ dei casi il sesso femminile e ha la sua massima incidenza nel periodo dell'adolescenza, con una prevalenza stimata tra lo 0.3 e lo $0.8 \%$. All'opposto dell'obesità, coinvolge maggiormente la popolazione del ceto medio-elevato. La severità di questa patologia è testimoniata dal tasso di mortalità, spesso per suicidio, che si aggira intorno al 5\% dopo 10 anni di malattia. Come per l'obesità, nella patogenesi dell'anoressia intervengono fattori genetici e ambientali. I primi sono fattori predisponenti e determinano la sensibilità del paziente e le sue reazioni emotive, condizionando così la sua suscettibilità alla malattia. I secondi agiscono come fattori scatenanti: sono molteplici e comprendono l'ambiente familiare (è stato stigmatizzato un atteggiamento prevaricante materno e troppo esigente paterno) e quello lavorativo (eccessive richieste produttive e conseguente ansia da prestazione), la cultura generale e individuale, i modelli estetici oggi vigenti, l'educazione alimentare. Questi fattori innescano quella distorsione/aberrazione comportamentale perseguita con esasperata determinazione che porterà il soggetto ad entrare e ad imprigionarsi dentro la malattia. La riduzione dell'introito calorico mediante restrizione alimentare spesso accompagnata a vomito autoindotto e l'aumento del 
dispendio energetico attraverso attività fisica intensiva e/o l'uso di farmaci, porterà ad una perdita di peso incontrollata e ad uno stato di denutrizione responsabile di serie di complicanze organiche (squilibri elettrolitici, aritmie cardiache, insufficienza epatica, osteoporosi) non raramente fatali.

Il trattamento di questa grave patologia non può ad oggi essere giudicato soddisfacente, considerato il tasso di guarigione $(50 \%)$, di solo miglioramento $(25 \%)$ e di passaggio alla cronicità o exitus $(25 \%)$. I risultati migliori si ottengono in centri specializzati in grado di praticare un trattamento multidisciplinare che veda coinvolte diverse competenze professionali comprendenti internisti, endocrinologi, psichiatri, psicologi e nutrizionisti. Purtroppo, esiste una grave carenza di strutture pubbliche dedicate ai disturbi del comportamento alimentare.

I lavori del convegno si chiuderanno con una Tavola Rotonda che porterà l'attenzione su importanti aspetti clinici dei disturbi del comportamento alimentare. Verranno infatti evidenziate le complicanze mediche che accompagnano spesso queste patologie e il ruolo determinante dell'intervento psicologico e nutrizionale nel loro trattamento.

Sono certo che al termine di questa giornata, grazie alle presentazioni di illustri conoscitori della materia, noi saremo arricchiti di conoscenze su queste due importanti patologie. 